\title{
Kaluza-Klein Type Model for the Structure of the Neutral Particle-like Solutions
}

\author{
Jacek Syska
}

Received: 15 February 2010 / Accepted: 1 June 2010 / Published online: 16 June 2010

(C) The Author(s) 2010. This article is published with open access at Springerlink.com

\begin{abstract}
The paper examines the geometrical properties of a six-dimensional Kaluza-Klein type model. They may have an impact on the model of the structure of a neutron and its excited states in the realm of particle physics. The statistical reason for the six-dimensionality and the stability of the solution is given. The derivation of the weak limit approximation of the general wave mechanical (quantum mechanical) approach, defined in the context of losing its self-consistency (here gravitational), is presented. The non self-consistent case for the Klein-Gordon equation is defined. The derivation of the energy of states and the analysis of the spin origin of the analyzed fields configuration is presented as the manifestation of both the geometry of the internal sample two-dimensional parametric space and kinematics of fields inside it. The problem of the departure from the (gravitational) self-consistent calculations of the metric tensor and of other fields of the configuration is discussed. The implementation of the model for the description of a neutron and its excited states, including their spins and energies, is given. The informational reason for the existence of the internal extra parametric space dimensions is proposed.
\end{abstract}

Keywords Wave mechanics of neutron · Self-consistent Kaluza-Klein field theory

\section{Introduction}

The paper presents the model of an elementary particle and particularly the model of the neutral nucleon and its excited states. Since the model is atomic-like I will give a concise reminder both of the history of the models of an atom and of a neutron which still overlap until the present day.

Possibly the first model of an atom in the 20th century was Kelvin's static one [1, 2] of a sphere of uniformly distributed positive electricity with embedded discrete electrons, followed closely by the Thomson's model of "the pudding with raisin muffins in it" [3].

\footnotetext{
J. Syska $(\bowtie)$

Department of Field Theory and Particle Physics, Institute of Physics, University of Silesia, Uniwersytecka 4, 40-007 Katowice, Poland

e-mail: jacek.syska@us.edu.pl
} 
Models also appeared at the end of the 19th century, stimulated by empirical facts suggesting the complexity of the structure of the atom and its composition from smaller components [4]. As a result, both the theoretical intuition and the empirical indications prompted the sense of the electron to Thomson. He disagreed with the idea of the planetary "point planets" model of the atom but probably was influenced by Maxwell's considerations on Saturn's rings [5]. Hence, contrary to Kelvin's model, the one by Thomson [3] is not static and one of its characteristics is the motion of a ring of $n$ negatively charged particles (electrons [6], as they were called by Stoney [7]), which are set within an uniformly charged sphere (with the radius of the order of $10^{-10} \mathrm{~m}$ ) having a number of particles which vary from ring to ring. The attractive force of the positively charged sphere and the repulsion of the negatively charged electrons arranged in a series of parallel rings were to guarantee the stability of the system. The strange characteristic of the model is that if the number of the negative particles is larger than 5, some of them must be set in the central position of the sphere yet not necessarily strictly in the center, or else the movement in the atom becomes unstable. This problem still arouses interest until the present day [8].

Thomson's atom has two types of vibrations: the first one connected with the movement of electrons along their orbits and the second one developing out of the distortion of a ring from its equilibrium circular shape in a particular spherical shell. Although the model does not explain the characteristic regularities of the observed spectral lines and the deflection of an electron when it passes through matter, it was regarded as a sufficient one by many physicists until the end of the first decade of the 20th century. This was due to the fact that he found a close relation between the occupation of the electrons inside rings formed in the concentric shells and the regularities inside the Mendeleev's periodic table. However, at the same time some scientists considered that the planetary model of the atom was more appropriate.

In 1920 Rutherford considered his model of the neutron in relation to the compressed hydrogen atom in the core of a star and presented his hypothesis on the structure of the neutron [9]. He perceived the neutron as an electron pressed into the interior of a proton hence his model of the neutron was more in line with Thomson's idea of the atom than of the planetary kind. In 1932 Chadwick experimentally confirmed Rutherford's hypothesis on the existence of the neutron.

In the period from 1925 to 1928 there emerged three formulations of quantum mechanics: Heisenberg's [10], Dirac's [11-14] and Schrödinger's [15-18]. They were developed nearly at the same time. The Schrödinger equation provides a direct representation of the mechanical system using the notion of the wave function for a particular state. Here the eigenstate functions form the orthogonal reference frame in the Hilbert space in which the wave function and its time evolution can be analyzed. The specific Schrödinger's substantial approach to the square modulus of the wave function gave the name 'wave mechanics' to his interpretation of quantum mechanics which is in line with de Broglie's theory of phase waves $[19,20]$.

Two questions about the main characteristics of the type of the models of an atom or nucleon built in these formalisms arise. Firstly, are they Thomson's with deep overlapping of constituent fields or planetary-like, for which the constituent fields do not overlap so deeply? On the surface, all of them carry some planetary features, especially if the Hamiltonians of the particular models are analyzed. This may be easily understood because in this respect they are classical mechanics descendants, yet in their main idea connected with the nonlocality of the wave function they are Thomson oriented, regardless of the fact whether the interpretation of the wave function is Schrödinger's or Copenhagen's. Indeed, in the atom the electron wave function is deeply embedded in the nucleus field. Secondly, what is the 
geometry of the manifold (space or space-time) which forms the background arena for the disputed configurations of fields? The common feature of Thomson's and Schrödinger's like models of the atom is the homogeneity of their space-time structure. We will come back to this problem in the passage below.

Now, let us return to the neutron. Since it seemed that quantum mechanics did not permit a consistent representation of the neutron as a bound state of the proton and electron, the before mentioned Rutherford's conception of the structure of the neutron has been rejected by the majority of physicists. Yet, until now attempts have been made to restore this model. For instance such attempts are connected with the construction of a covering of quantum mechanics known as hadronic mechanics [21-26] for the specific objective of achieving a consistent quantitative treatment of nonlocal, nonlinear and nonpotential effects in deep wave-overlappings of particles at short distances. As far as the quantum models of the neutron are considered, the most popular is the quantum field theory version of the bag model. Setting aside the difficulties of this model with the determination of the spin of the nucleon we give some literature references $[27,28]$ only citing after [29]: “ $\ldots$ all spin parts [of the nucleon] have to add to $\frac{1}{2}$ which is incredible in the light of the present day experiments. This may indicate that some underlying symmetries, unknown at present, are playing a role in forming the various contributing parts such that the final sum rule gives the fermion $\frac{1}{2}$ value".

Another story is partly connected with the name of Riemann. In his lecture entitled "On the hypotheses that lie at the foundations of geometry" [30], given in 1854, he emphasized that the truth about space has to be discovered ${ }^{1}$ from physical experience. The clue is that he noted that the geometry of space could be highly irregular at very small distances, yet appearing smooth at the observed ones. He wrote: "Space [in the large] if one ascribes to it a constant curvature, is necessarily finite, provided only that this curvature has a positive value, however small... It is quite conceivable that the geometry of space in the very small does not satisfy the axioms of [Euclidean] geometry...".

But the story of the atom and nucleon may be told and retold (at least up to now) in many ways [31]. Not that all of them are right. The latest attractive model of the (hydrogen) atom, based on the isomorphism between Maxwell and Dirac formalisms (or rather on optics-mechanics isomorphism called Hamilton's analogy), is connected with the name of Sallhofer [32]. He worked out the formal mathematical strong similarity (I would not call it identity), in the Minkowski space, between electrodynamics and wave mechanics by means of which he proved that the hydrogen atom might be seen as a pair of mutually refracting electromagnetic waves. Previously this similarity was pointed out with amusement by Sakurai [33].

Below the model of a neutral nucleon will be presented which is more planetary (fields do not overlap very deeply) and incorporates the Riemann's idea of space. The metric of the underlying space-time was previously obtained in [34-36] where a six-dimensional KaluzaKlein type model at the classical level is considered. In them the static spherically symmetric solution to the coupled six-dimensional Einstein and Klein-Gordon equations was derived in the presence of the basic massless dilaton field $\varphi$, which variation in the radial direction compensates self-consistently for the curvature. The dilatonic field $\varphi$ forms a kind of ground field for the self-field of gravitation, the notions of which were discussed elsewhere [37-49]. The solutions presented in $[34,35]$ are parameterized by one parameter $A$ which has similar

\footnotetext{
${ }^{1}$ I do not agree with this point of view, but definitely the truth about space is consistent with physical experience. I consider the physical world to be perhaps at least six-dimensional with at least four of these dimensions to be of the space-time origin.
} 
dynamical consequences as mass [50] $M=A c^{2} /(2 G)$, i.e. its existence would be perceived by an observer in the same way as invisible mass which could be the extended "center" of a particle. Because the solution is horizon free hence it is fundamentally different from the four-dimensional Schwarzschild one.

The motivations which lie behind the six-dimensionality of the space-time and the stability of the solutions are presented in Sects. 1.1 and 2.1, respectively. In Sect. 3 the weak limit approximation of the general wave mechanical approach for the new scalar field inserted into the "reference" dilatonic background is defined. In Sect. 3.4 the analysis of the phenomenon of the breaking of the scale invariance of the coupled Einstein and Klein-Gordon equations is given. It will result from the lower symmetry of the self-consistent solution of these equations, where the original dilatonic field $\varphi$ is the Goldstone one. In Sect. 4.1 the neutral particle-like solution, which could be called a 'neutron', is obtained. In Sect. 4.3 the results connected with the gravitational bounding of the 'neutron' and its excited states are presented. This means that, as in the case of the 'neutron', the formalism makes it possible to describe the half-integer spin particle with the use of the dynamics of the scalar field in the six-dimensional space-time. An example of the integer spin ' $\rho_{o}$ ' neutral meson is also given. Finally, a new concept on the simultaneous variety of the dimensions of parametric spaces for structures, even more complicated than spinors, also emerges. This feature of the model connected with the possible observation of several large extra dimensions ought to be tested in accelerator experiments.

\subsection{The Motivations for Extra Dimensions}

The idea of the six-dimensional space-time re-enters the physics occasionally [51-53]. Yet the motivations for choosing the six-dimensional models were diverse. For instance in $[51,52]$ Nishino, Salam and Sezgin suggested that one may obtain the fermion spectrum in four-dimensions within the framework of $D=6, N=2$ Kaluza-Klein supergravity. A sixdimensional model of the Kaluza-Klein theory was also previously investigated by Mańka and Syska [48, 53], and by Ivashchuk, Melnikov and Bronnikov [54-57]. Recently Sparling [58] has followed the concept of $(3+3)$ dimensional structure of the space-time [59-61]. Unfortunately, models described in [58-61] contain the time component with two additional time dimensions.

However, still more closely to the Standard Model modelling, there is a way to extend the four-dimensional space-time to the multi-dimensional one via the Fisherian statistical analysis. Few years ago Frieden proved [62-71] that the Fisher information channel capacity is the statistical ancestor of the kinematical part of the well known field theory models. Using the channel capacity notion, which is inherently connected with the internal parametric space of the sample (of positions collected by the particle alone), and two informational principles, the structural and the variational one, Frieden and Soffer [62-70] obtained the Klein-Gordon equation of motion with the proper relativistic dispersion relation. ${ }^{2}$ The structural information principle has been recently proven in $[72,73]$. The channel capacity, the trace of the Fisher information matrix, conveys in our case the Fisher information on the quality of the (unseen) position parameters ${ }^{3}$ estimation, being the upper bound for the Stam's information, which is the total intrinsic accuracy of the model. The point is that the channel capacity which has turned out to be the kinematical part of the action of any field theory model, is

\footnotetext{
${ }^{2}$ They obtained the proper structural equation of motions for the Maxwell electromagnetic field, for the Dirac field and for the gravitational field also [62-71].

${ }^{3}$ They have nothing to do with the so called classical hidden variables [72].
} 
additive both in the Minkowskian $v=0,1,2,3$ and the sample $n=1,2, \ldots, \aleph$ (parametric) indices [62-70]. This enables the partition of the channel capacity $I$ into two parts, one for the four-dimensional space-time and the other for the inner parametric space of the sample whose dimension depends on the spinorial representation of the field [62-73]. When we note by $x_{n}^{\nu}=y_{n}^{\nu}-\theta_{n}^{v}$ the added fluctuations of the data $y_{n}^{v}$ (collected by the system alone) from the expectation positions [62-70] $\theta_{n}^{v} \equiv \theta^{v} x_{n}$, then it can be proven that it takes the following form:

$$
\begin{aligned}
I & =4 \sum_{n=1}^{\aleph} \int d^{4} y_{n}\left(\frac{\partial q_{n}\left(y_{n}^{v}\right)}{\partial \theta_{n}^{v}} \frac{\partial q_{n}\left(y_{n}^{v}\right)}{\partial \theta_{v n}}\right) \\
& \equiv \int d^{4} y_{n} \mathcal{L}\left(\eta_{\mu \nu}, q_{n}\left(y_{n}^{v}\right)\right) \\
& =\int d^{4} x \mathcal{L}_{4}\left(g_{\mu \nu}, \varphi_{n}\left(x^{\nu}\right)\right)+\int d^{\aleph} x \mathcal{L}_{\aleph}\left(g_{m n}, \varphi_{\aleph}\left(x_{n}\right)\right),
\end{aligned}
$$

where $q_{n}\left(y_{n}^{v}\right)$ are the original field amplitudes ${ }^{4}$ in the sample [62-74], $\eta_{\mu \nu}$ is the Minkowskian metric, $x_{n}^{\nu}$ are the original Fisherian variables and $x_{n}$ are the parameters. The index $n$ has been dropped from the first integral on the RHS of (1) as for the given $v$ the range of all $x_{n}^{v}$ is the same. The amplitudes are factorized as follows $q_{n}\left(y_{n}^{\nu}\right)=f \varphi_{n}\left(x^{\nu}\right) \varphi_{\aleph}\left(x_{n}\right)$, where $f$ is a proportionality constant and the metric tensor $g_{M N}(M, N \equiv(\mu, v=0, \ldots, 3 ; m, n=1, \ldots, \aleph))$ is obtained effectively from this procedure [75]. Factor 4 in the kinematical form of $I$ signifies the Fisher information origin of the action, yet it does not enter into the equation of motion [62-70] as it is also factored out from the structural information [72, 73].

For the complex scalar field the dimension $\aleph$ of the extra parametric space is equal to $\aleph=2$. In this way the six-dimensionality of the space having the mixed space-time and parametric character is chosen. Hence the model with the internal space geometry of the two-dimensional parametric space parameterized by two angles will be presented. This provides the geometry of internal space, which in Sects. 3 and 4 enables us to describe the spinorial field. In [34, 35], Biesiada, Mańka and Syska showed that the six-dimensionality of this space-time enables the self-consistency of the background solution of the coupled Einstein and Klein-Gordon equations with the internal parametric space, compactified in a non-homogeneous manner. For the sake of clarity, the summary of [34, 35] is presented below in Sect. 2. In Sects. 3 and 4 the model will raise a new issue on the wave-mechanical analysis of the possible general structure of a particle. In Sect. 4 its application to the description of the 'neutron' and its excited states is presented. In general, statistical models which one can obtain in this way are of the Kaluza-Klein type with four-dimensional spacetime and $\aleph$-dimensional internal parametric space.

\section{The Geometry of the Space-Time}

In [34-36] a $(1+5)$ six-dimensional field theory has been considered (see also [77]) which comprises the gravitational self field described by a metric tensor, $g_{M N}$, and a real massless "basic" scalar (dilatonic) field, $\varphi$. We have decomposed the action into two parts:

\footnotetext{
${ }^{4}$ After squaring each one of $q_{n}\left(y_{n}^{v}\right)$ and multiplication, the likelihood of the sample is calculated.
} 


$$
\begin{aligned}
\mathcal{S} & =\mathcal{S}_{E H}+\mathcal{S}_{\varphi} \\
& =\int d^{6} x \sqrt{-g} \frac{1}{2 \kappa_{6}} \mathcal{R}+\int d^{6} x \sqrt{-g} \frac{-g_{M N}}{2} \partial^{M} \varphi \partial^{N} \varphi,
\end{aligned}
$$

where $S_{E H}$ is the Einstein-Hilbert action and $S_{\varphi}$ is the action for a real massless scalar (dilatonic) field with the Lagrangian density equal to $\mathcal{L}_{\varphi}=-\frac{1}{2} g_{M N} \partial^{M} \varphi \partial^{N} \varphi$. In (2) $g=\operatorname{det} g_{M N}$ denotes the determinant of the metric tensor, $\mathcal{R}$ is the curvature scalar of the six-dimensional (in general curved) space-time, and $\kappa_{6}$ denotes the coupling constant of the six-dimensional theory, analogous to the familiar Newtonian gravity constant.

By extremalizing the action given by (2) we obtain the Einstein equations

$$
G_{M N}=\kappa_{6} T_{M N},
$$

where $G_{M N}=R_{M N}-\frac{1}{2} g_{M N} \mathcal{R}$ is the Einstein tensor, $R_{M N}$ is the six-dimensional Ricci tensor, $\mathcal{R}$ is the curvature scalar and $T_{M N}$ is the energy-momentum tensor of a real scalar (dilatonic) field $\varphi$ which is given by

$$
T_{N}^{M}=\partial_{N} \varphi \frac{\partial \mathcal{L}_{\varphi}}{\partial\left(\partial_{M} \varphi\right)}-\delta_{N}^{M} \mathcal{L}_{\varphi}
$$

Variation of the total action $\mathcal{S}$ with respect to the field $\varphi$ gives the Klein-Gordon equation

$$
\square \varphi \equiv-\frac{1}{\sqrt{-g}} \partial_{M}\left(\sqrt{-g} g^{M N} \partial_{N}\right) \varphi=0,
$$

where $g^{M N}$ is the tensor dual to $g_{M N}$.

Now consider the six-dimensional space-time which is a topological product of the curved four-dimensional physical space-time (with the metric $g_{\alpha \omega}, \alpha, \omega=0,1,2,3$ ) and the compactified parametric space (with the metric $\left.g_{h e}, h, e=5,6\right) .^{5}$ Therefore the metric tensor can be factorized as

$$
g_{M N}=\left(\begin{array}{cc}
g_{\alpha \omega} & 0 \\
0 & g_{h e}
\end{array}\right)
$$

The four-dimensional diagonal part is assumed to be that of a spherically symmetric geometry

$$
g_{\alpha \omega}=\left(\begin{array}{cccc}
e^{\nu(r)} & & & \\
& -e^{\mu(r)} & 0 & \\
& 0 & -r^{2} & \\
& & & -r^{2} \sin ^{2} \Theta
\end{array}\right),
$$

where $v(r)$ and $\mu(r)$ are (at this stage) two arbitrary functions. Analogously, we take the two-dimensional internal part to be

$$
g_{\text {he }}=\left(\begin{array}{cc}
-\varrho^{2}(r) \cos ^{2} \vartheta & 0 \\
0 & -\varrho^{2}(r)
\end{array}\right) .
$$

\footnotetext{
${ }^{5}$ This reflects the generalization of the principal bundle to the case of the statistical space and the sample space-time as the base space. The axes in the parametric space span the coordinate system in a subspace of the statistical space.
} 
The six-dimensional coordinates $\left(x^{M}\right)$ are denoted by $(t, r, \Theta, \Phi, \vartheta, \varsigma)$ where $t \in[0, \infty)$ is the usual time coordinate, $r \in[0, \infty), \Theta \in[0, \pi]$ and $\Phi \in[0,2 \pi)$ are the familiar threedimensional spherical coordinates in the macroscopic space; $\vartheta \in[-\pi, \pi)$ and $\varsigma \in[0,2 \pi)$ are coordinates in the two-dimensional parametric space and $\varrho \in(0, \infty)$ is the "radius" of this internal space. We assume that $\varrho(r)$ is the function of the radius $r$ in the external threedimensional space. The internal space is a 2-dimensional parametric space with $r$-dependent parameter $\varrho(r)$. Using (7)-(8), we can calculate the components of the Ricci tensor. They are given in Appendix A.

Now we seek for a solution of the Einstein equations (see (3)) with the Ricci tensor given in Appendix A by (71)-(74), with $v(r)=\mu(r)$, and with the following boundary conditions

$$
\begin{aligned}
& \lim _{r \rightarrow \infty} v(r)=\lim _{r \rightarrow \infty} \mu(r)=0, \\
& \lim _{r \rightarrow \infty} \varrho(r)=d=\text { constant } \neq 0,
\end{aligned}
$$

which at the spatial infinity reproduces the flat external four-dimensional Minkowski spacetime and the static parametric space of "radius" $d$ which is of the order calculated in Sect. 4. We also suppose that the scalar field $\varphi$ is the function of the radius $r$ alone, $\varphi=\varphi(r)$, and we impose the following boundary condition for the scalar field $\varphi$ :

$$
\lim _{r \rightarrow \infty} \varphi(r)=0
$$

which supplements boundary conditions (9) for the metric components.

By virtue of (4) and (2) it is easy to see that the only nonvanishing components of the energy-momentum tensor are

$$
-T_{r}^{r}=T_{t}^{t}=T_{\Theta}^{\Theta}=T_{\Phi}^{\Phi}=T_{\vartheta}^{\vartheta}=T_{\varsigma}^{\varsigma}=\frac{1}{2} g^{r r}\left(\partial_{r} \varphi\right)^{2} .
$$

Consequently, it is easy to verify that the self-consistent solution of the coupled Einstein (3) and Klein-Gordon (5) equations is

$$
\begin{aligned}
& v(r)=\mu(r)=\ln \left(\frac{r}{r+A}\right), \quad \varrho(r)=d \sqrt{\frac{r+A}{r}}, \\
& \varphi(r)= \pm \sqrt{\frac{1}{2 \kappa_{6}}} \ln \left(\frac{r}{r+A}\right) .
\end{aligned}
$$

Hence it results that the only nonzero component of the Ricci tensor (see (71)-(74) in Appendix A) is $R_{r}^{r}$. So the curvature scalar $\mathcal{R}$ is equal to

$$
\mathcal{R}=R_{r}^{r}=\frac{A^{2}}{2 r^{3}(r+A)},
$$

where $A$ is the real constant, with the dimensionality of length, whose value is to be taken from the observation of each particular system (but see Sect. 4). We notice that all of the six diagonal Einstein equations (3) have shrunk to just one

$$
\frac{1}{2} \mathcal{R}=\kappa_{6} T_{r}^{r}
$$


Now we can rewrite the metric tensor in the form

$$
\begin{aligned}
g_{M N}= & \operatorname{diag}\left(\frac{r}{r+A},-\frac{r}{r+A},-r^{2},-r^{2} \sin ^{2} \Theta,\right. \\
& \left.-d^{2} \frac{r+A}{r} \cos ^{2} \vartheta,-d^{2} \frac{r+A}{r}\right)
\end{aligned}
$$

with its determinant equal to

$$
g=\operatorname{det} g_{M N}=-\left(d^{2} r^{2} \sin \Theta \cos \vartheta\right)^{2} .
$$

To summarize we notice that the real massless "basic" free scalar field $\varphi(r)$ (see (13)) can be the source of the nonzero metric tensor as in (16). Only when the constant $A$ is equal to zero, the solutions (12)-(13) become trivial and the six-dimensional space-time is Ricci flat.

It is worth noting that since (with the solutions given by (12) and (13)) the components $R_{\vartheta}^{\vartheta}$ and $R_{\varsigma}^{\varsigma}$ of the Ricci tensor (Appendix A) are equal to zero for all values of $A$, the parametric space is always Ricci flat. However, we must not neglect the parametric space because its "radius" $\varrho$ is a function of $r$ and the two spaces, external and parametric, are therefore "coupled". Only when $A=0$ are these two spaces "decoupled", and the fourdimensional space-time becomes Minkowski flat. ${ }^{6}$

\subsection{Stability of the Background Solution}

The self consistent solution given by (13) and (16) of the coupled Einstein and Klein-Gordon equations is unique. To answer the question on the stability of this self-consistent gravitydilatonic configuration let us calculate its energy:

$$
E_{g+\varphi}=\int_{V} d^{5} x \sqrt{-g}\left(G^{t t}+\kappa_{6} T^{t t}\right)
$$

where using (11)-(17) we obtain:

$$
E_{g+\varphi}=-2 \int_{V} d^{5} x \sqrt{-g} g^{t t} \frac{\mathcal{R}}{2}=-2 Q
$$

with

$$
\begin{aligned}
Q & =8 d^{2} \pi^{2} \lim _{\varepsilon \rightarrow 0^{+}} \int_{\varepsilon}^{\infty} \frac{A^{2}}{r^{2}} d r \\
& =(2 \pi d)\left(4 \pi A^{2}\right) \lim _{\varepsilon \rightarrow 0^{+}} \frac{d}{\varepsilon} .
\end{aligned}
$$

The integral of $1 / r^{2}$ does not converge on $(0, \infty)$. Hence (19) means that the self consistent solution (13) and (16) cannot be obtained from $A \rightarrow 0$ finite energy (even in the limit $E_{g+\varphi} \rightarrow 0$ ) convergent solutions around $E_{g+\varphi}=0$ corresponding to $A=0$.

If we depart from the self consistent solution then a similar result can be obtained from the information analysis in the following way. From the notion of the Fisher information

\footnotetext{
${ }^{6}$ When $A$ is not equal to zero, our four-dimensional external space-time is curved. Its scalar curvature $\mathcal{R}_{4}$ can be given by (14), i.e. $\mathcal{R}_{4}=\mathcal{R}=\frac{A^{2}}{2 r^{3}(r+A)}$. 
[62-70], we notice that $d$ is (for $r \rightarrow \infty$, see (12)) the characteristic dimension of the parametric space, i.e. the radius of the expected value of the position of the system. In the Frieden [62-70] analysis $r$ is the added fluctuation to $d$. Hence if we assume that the fluctuation $r$ is not smaller than the expected value $d$ then the physical limit for the fluctuation $r$ is $\varepsilon \approx d$. In (20) the cutoff $\varepsilon=d$ can be taken which leads to $Q=Q_{\text {cut }}=(2 \pi d)\left(4 \pi A^{2}\right)$. Therefore $Q_{c u t}$ is finite and its value could be even small in contradistinction to the infinite value obtained in (20) for $\varepsilon \rightarrow 0$ in the self consistent case. Hence, we see that the unique self consistent solution given by (13) and (16) cannot be destabilized to yield any other.

Let us also notice that in (18) the partition of $E_{g+\varphi}$ into two parts can be obtained by using the Fisher information formalism developed for physical models by Frieden [62-70]. According to [62-70], $G^{t t}$ is connected with the Fisherian kinematical degrees of freedom of the gravitational configuration whereas $T^{t t}$ with its structural degrees of freedom.

To conclude this section it is worth noting that the metric given by (16), with the dilatonic field given by (13), serves, under further conditions discussed below, as a background fields configuration. This metric appears in the equation of motions for all new fields which weakly enter the system.

\section{The Quantum Implication-Klein-Gordon Equation. Wave-mechanical Approach and Weak Interaction Limit}

Until now the calculations were fully self-consistent. The model presented below adds a new scalar field to the system. Yet, since it procures big analytical complications the model will stop being fully self-consistent. This means among others that we will decline from making the self-consistent correction of the metric (16) obtained in Sect. 2. Therefore the following calculations are made totally on the basis of the wave-mechanical approach.

Let us investigate the Klein-Gordon relativistic wave equation for the motion of a scalar particle with a wave function $\phi$ and mass $m$ (for $m^{2}<0$ it would be a 6-dimensional tachion),

$$
\frac{1}{\sqrt{-g}}(i \hbar) \partial_{M}\left(\sqrt{-g} g^{M N}(i \hbar) \partial_{N} \phi\right)-m^{2} c^{2} \phi=0,
$$

in the six-dimensional space-time given by the central gravitational field described by the metric, $g_{M N}$, given by (16) with its determinant given by (17). This metric tensor is used in the above Klein-Gordon equation as the background metric only. Hence we neglect the modification of the Einstein equations. Therefore a particle described by the $\phi$ wave function moves without changing the metric $g_{M N}$ in reciprocal action. This is called the wavemechanical approach. But to make our calculations self-consistent by the inclusion of the Einstein equations coupled to Klein-Gordon equation, we should follow the pattern of the previous section for all scalar fields (particles) which have the mass $m$ and are inherently tied up by strong interaction (compare Sect. 4.3.1). This procedure should give the selfconsistent change of the metric tensor $g_{M N}$.

We incorporate $[48,49]$ the so-called "natural interpretation" of the wave equation ${ }^{8}$ with a particle as an oscillating substance described by the wave function $\phi$ which is deformable according to (21).

\footnotetext{
${ }^{7}$ Also the inverse of the Fisher information gives according to the Cramer-Rao inequality [62-70] the lower bound on the square error $\left(\propto r^{2}\right)$ of the estimator of $d$.

${ }^{8}$ Then we do not have any problems with possible negative values of probability density which is the inherent property of the probabilistic interpretation of quantum mechanics for the Klein-Gordon equation [78].
} 
Using (16) and (17), we can rewrite the Klein-Gordon equation as follows:

$$
\begin{aligned}
& \sin \Theta \cos \vartheta\left(\frac{r+A}{r} r^{2} \frac{\partial}{c \partial t}\left(\frac{\partial \phi}{c \partial t}\right)-\frac{\partial}{\partial r}\left(r^{2} \frac{r+A}{r} \frac{\partial \phi}{\partial r}\right)\right) \\
& -r^{2} \cos \vartheta\left(\frac{\partial}{\partial \Theta}\left(\sin \Theta \frac{1}{r^{2}} \frac{\partial \phi}{\partial \Theta}\right)+\sin \Theta \frac{1}{r^{2} \sin ^{2} \Theta}\left(\frac{\partial^{2} \phi}{\partial \Phi^{2}}\right)\right) \\
& -\frac{r^{2}}{d^{2}} \sin \Theta\left(\frac{r}{r+A} \frac{\partial}{\partial \vartheta}\left(\cos \vartheta \frac{\partial \phi}{\partial \vartheta}\right)+\cos \vartheta \frac{r}{r+A} \frac{\partial^{2} \phi}{\partial \varsigma^{2}}\right) \\
& +r^{2} \sin \Theta \cos \vartheta \frac{m^{2} c^{2}}{\hbar^{2}} \phi=0 .
\end{aligned}
$$

In order to isolate the time dependence, the standard procedure of the separation of variables is performed with the following factorization of $\phi$

$$
\phi(\mathbf{r}, \vartheta, \varsigma, t)=u(\mathbf{r}, \vartheta, \varsigma) e^{-i \frac{E t}{\hbar}} .
$$

In this way, we obtain the set of stationary states. As the result of further separation in the three-dimensional spherical coordinates $\mathbf{r}=(r, \Theta, \Phi)$ of the macroscopic space and in $(\vartheta, \varsigma)$ coordinates of the parametric two-dimensional space described by the factorization

$$
u(\mathbf{r}, \vartheta, \varsigma)=u_{r}(r) Y_{L M}(\Theta, \Phi) y_{l \tilde{\mathrm{m}}}(\vartheta, \varsigma)
$$

we obtain the "almost" familiar radial Klein-Gordon wave equation [78]

$$
\begin{aligned}
& \frac{r}{r+A}\left\{-\frac{1}{r^{2}} \frac{\partial}{\partial r}\left(\frac{r+A}{r} r^{2} \frac{\partial u_{r}}{\partial r}\right)+\frac{L(L+1)}{r^{2}} u_{r}\right\} \\
& =\frac{1}{\hbar^{2} c^{2}}\left\{E^{2}-m^{2} c^{4} \frac{r}{r+A}-\lambda_{d} \frac{\hbar^{2} c^{2}}{d^{2}}\left(\frac{r}{r+A}\right)^{2}\right\} u_{r}
\end{aligned}
$$

with the angular equation in the macroscopic space which defines states with a definite three-dimensional angular momentum

$$
\begin{aligned}
& -\hbar^{2}\left[\frac{1}{\sin \Theta} \frac{\partial}{\partial \Theta}\left(\sin \Theta \frac{\partial}{\partial \Theta}\right)+\frac{1}{\sin ^{2} \Theta} \frac{\partial^{2}}{\partial \Phi^{2}}\right] Y_{L M} \\
& =L(L+1) \hbar^{2} Y_{L M},
\end{aligned}
$$

and the angular equation in the parametric space with a definite internal angular momentum

$$
\frac{1}{\cos \vartheta} \frac{\partial}{\partial \vartheta}\left(\cos \vartheta \frac{\partial}{\partial \vartheta} y_{l \tilde{\mathrm{m}}}\right)+\frac{1}{\partial \varsigma}\left(\frac{\partial}{\partial \varsigma} y_{l \tilde{\mathrm{m}}}\right)=-\lambda_{d} y_{l \tilde{\mathrm{m}}},
$$

where $\lambda_{d}$ is the main internal angular quantum number. In (26) functions $Y_{L M}$ are the spherical harmonics (normalized angular momentum eigenfunctions) with $L=0,1,2, \ldots$ and $M=0, \pm 1, \ldots, \pm L$. Similarly $y_{l \tilde{\mathrm{m}}}$ are the periodic functions of the inner angles $\vartheta$ and $\varsigma$. As the result of further separation of (27) in the internal coordinates described by the factorization

$$
y_{l \tilde{\mathrm{m}}}(\vartheta, \varsigma)=g_{l}(\vartheta) h_{\tilde{\mathrm{m}}}(\varsigma)
$$


we obtain

$$
\frac{1}{\cos \vartheta} \frac{\partial}{\partial \vartheta}\left(\cos \vartheta \frac{\partial}{\partial \vartheta} g_{l}\right)+k_{l} g_{l}=0,
$$

where $k_{l}$ is the internal $\vartheta$-angle quantum number of the parametric space geometry (see Sect. 3.2).

\subsection{The Case with $\lambda_{d}=0$ and $m=0$ and Its Difference from Others}

It is not difficult to verify that for $\lambda_{d}=0$ and $m=0$ the only solution of the stationary Klein-Gordon equation (25) alone, vanishing in infinity (although not normalizable), exists for $E=0$ and $L=0$. For the radial part of $\phi$ this solution has the following form (compare (13) for $\varphi(r))$ :

$$
u_{r}(r)=C \ln \left(\frac{r}{r+A}\right) \text {, }
$$

where $C$ is a constant. In this way we formally obtain $[34,35]$ up to $C$ the same solution which follows from the classical Klein-Gordon equation for the dilaton field (see (5)). Yet now the field is a scalar one, hence the energy of the configuration (30) is positive and equals:

$$
\int_{V} d^{5} x \sqrt{-g} T_{\phi}^{t t}=2 C^{2} Q
$$

where $T_{\phi}^{t t}$ is the $t t$ component of the energy-momentum tensor of the scalar field $\phi$. Integral $Q$ does not converge and is given by (20). ${ }^{9}$ However, fortunately here, we may recall the selfconsistent calculations for fields which were previously introduced, i.e. the dilaton $\varphi$ and metric $g_{M N}$ ones, with the now added scalar field $\phi$, which we for the time being assume to be given by the configuration (30). The coupled Einstein and Klein-Gordon field equations for all these fields have to be solved. Now, the case with $\lambda_{d}=0, m=0, E=0$ is the only one for which the value of $\hbar$ in (25) is irrelevant as then the RHS of (25) becomes equal to zero. Therefore in this case the energy-momentum tensor for the massless scalar field enters into the RHS of Einstein equations (3) in the same way as the dilaton field, differing only in sign. Hence the RHS of Einstein equations (3) becomes equal to zero leading to Ricci flat solution of Einstein equations. Therefore the discussed new configuration of all fields, $g_{M N}, \varphi$ and $\phi$, does not describe the gravitational bound state, contrary to the case of the metric-dilaton field configuration given by (13) and (16) alone. Hence the configuration given by (30) for $m=0$ and $\lambda_{d}=0$ does not appear. ${ }^{10}$

However, a similar conclusion would not be true for $\lambda_{d}>0$ even for $m=0$. Indeed, in all these cases the Planck constant $\hbar$ in (25) becomes important and leads to the appearance of the energy-momentum tensor for the scalar field, $T_{\phi N}^{M}\left(\hbar^{2}\right)$, which is of the order

\footnotetext{
${ }^{9}$ As before in (20), the integral of $1 / r^{2}$ in $Q$ does not converge on $(0, \infty)$. Hence the solution (30) obtained for $E=0, m=0, \lambda_{d}=0$ and $L=0$ cannot be obtained even from the solutions around $E=0, m=0$ (i.e. in the limit $E \rightarrow 0, m \rightarrow 0$ ) for which the LHS of (31) converges.

${ }^{10}$ We may say that solution (30) of (25) is in this case as "macroscopic" as the dilatonic configuration (13) leading to the zeroing on the RHS of the Einstein equations (3). In other words: The only Thomson like solution of the scalar configuration (30), which by definition cannot be treated as a small perturbation of the metric-dilaton configuration, does not appear at all.
} 
of $\hbar^{2}$. Hence $T_{\phi N}^{M}\left(\hbar^{2}\right)$ constitutes a tiny quantity in comparison to the energy-momentum tensor for the dilaton field, $T_{N}^{M}\left(\hbar^{0}\right)$ (see (11) and (13)), which appears on the RHS of Einstein equations (3). This means that in the first approximation we may treat the solutions of (25) for $\lambda_{d}>0$ as leading to small perturbations of the background metric (16), ignoring its self-consistent modification. In other words, the backreaction of $\phi$ is negligible in this case.

\subsection{Further Steps for $k_{l}=l(l+1)$}

If the internal $\vartheta$-angle quantum number of the parametric space geometry is set to $k_{l}=$ $l(l+1)$ then $g_{l}(\vartheta)=P_{l}(\sin \vartheta), l=0,1,2, \ldots$, are the Lagrange polynomials which solve (29) and hence the second factor on the RHS of (28) satisfies the following equation (see (27))

$$
\frac{1}{\partial \varsigma}\left(\frac{\partial}{\partial \varsigma} h_{\tilde{\mathrm{m}}}\right)=-\left[-l(l+1)+\lambda_{d}\right] h_{\tilde{\mathrm{m}}}
$$

The condition of continuity of functions $h_{\tilde{\mathrm{m}}}(\varsigma)$ for $\varsigma \in[0,2 \pi)$ together with $h_{\tilde{\mathrm{m}}}(0)=$ $h_{\tilde{\mathrm{m}}}(2 \pi)$ give

$$
\begin{aligned}
h_{\tilde{\mathrm{m}}}(\varsigma) & =e^{ \pm i \sqrt{-l(l+1)+\lambda_{d}} \varsigma} \\
& =e^{i \tilde{\mathrm{m}} \varsigma}, \quad \tilde{\mathrm{m}}=0, \pm 1, \pm 2, \ldots,
\end{aligned}
$$

where $\tilde{\mathrm{m}}$ is the internal magnetic quantum number. Now, from the above equation we can notice that $\lambda_{d}$ is a quantum number which chooses the ladder of values:

$$
\lambda_{d}=l(l+1)+\tilde{\mathrm{m}}^{2}=0,1,2,3,4,6,6,7,9,10, \ldots
$$

hence $\lambda_{d} \geq 0$. For example $\lambda_{d}=1$ in two cases: $(l=0$ and $\tilde{\mathrm{m}}=1)$ and $(l=0$ and $\tilde{\mathrm{m}}=-1)$. Let us also notice that under the assumption of $m=0$ the case with $\lambda_{d}=0$ was previously excluded (Sect. 3.1). ${ }^{11}$

\subsection{The Weak Interaction Limit}

Let us focus on the case of the weak limit which means that we are far away $(r \gg A)$ from the central core of the dylatonic field given by (13). At first let us rewrite (25) as follows

\footnotetext{
${ }^{11}$ We will see in Sect. 4.3 that only the mass $m=0$ satisfies the two physical requirements, the first one of stability and the second one of the proper value of the total spin of the 'nucleon'. Hence eventually only $\lambda_{d}>0$ will be possible. 


$$
\begin{aligned}
& \frac{A}{r^{2}}\left(\frac{r}{r+A}\right) \frac{\partial u_{r}}{\partial r}-\frac{1}{r^{2}} \frac{\partial}{\partial r}\left(r^{2} \frac{\partial u_{r}}{\partial r}\right) \\
& \quad+\left(\frac{r}{r+A}\right) \frac{L(L+1)}{r^{2}} u_{r} \\
& =\frac{1}{\hbar^{2} c^{2}}\left\{E^{2}-m^{2} c^{4}\left(\frac{r}{r+A}\right)-\lambda_{d} \frac{\hbar^{2} c^{2}}{d^{2}}\left(\frac{r}{r+A}\right)^{2}\right\} u_{r} .
\end{aligned}
$$

Now, asymptotically (i.e. $r \rightarrow \infty$ ) the first and third term of the LHS of the above equation could be omitted as they are of smaller order than the others. Yet, the third term, with the orbital angular momentum $L$, will be preserved as it is important in the angular momentum analysis. From the point of view of the energies ${ }^{12}$ of the excited states (Sect. 4) it would be better in the limit $r \rightarrow \infty$ to omit consistently all terms which are proportional to $\frac{1}{r^{2}}$ since leaving only the one with $L$ leads, in light of the omission of the first one, to the appearance of nonphysical states.

Nevertheless let us omit the first term in (35) only which then, after introducing the variable $x=A / r$, could be rewritten as follows:

$$
\begin{aligned}
& -\frac{1}{A^{2}} x^{4} \frac{\partial^{2} u_{r}}{\partial x^{2}}+\frac{x^{2}}{1+x} \frac{L(L+1)}{A^{2}} u_{r} \\
& =\frac{1}{\hbar^{2} c^{2}}\left(E^{2}-m^{2} c^{4} \frac{1}{1+x}-\lambda_{d} \frac{\hbar^{2} c^{2}}{d^{2}}\left(\frac{1}{1+x}\right)^{2}\right) u_{r} .
\end{aligned}
$$

Now, let us expand the coefficients in (36) at point $x=x_{0}=0$ (i.e. $r \longrightarrow \infty$ ) to the second order in $x$. We obtain

$$
\begin{aligned}
-\hbar^{2} c^{2} & {\left[\frac{x^{4}}{A^{2}} \frac{\partial^{2} u_{r}}{\partial x^{2}}-\left(p_{0}+p_{1} x+p_{2} x^{2}\right) \frac{L(L+1)}{A^{2}} u_{r}\right] } \\
= & \left\{E^{2}-\left(\mu_{0}+\mu_{1} x+\mu_{2} x^{2}\right) m^{2} c^{4}\right. \\
& \left.-\left(\lambda_{0}+\lambda_{1} x+\lambda_{2} x^{2}\right) \lambda_{d}\left(\frac{\hbar c}{d}\right)^{2}\right\} u_{r},
\end{aligned}
$$

where the coefficients of the expansions and their limits for $x_{0}=0(r \longrightarrow \infty)$ are given in Appendix B.

Finally, after coming back to variable $r$ we obtain for $r \gg A$ the radial part of the KleinGordon-like equation, written in the spherical coordinates as follows

$$
\begin{gathered}
-\frac{1}{r^{2}} \frac{\partial}{\partial r}\left(r^{2} \frac{\partial u_{r}}{\partial r}\right)+\left(U_{R e p}-U_{A t t r}\right) u_{r} \\
=\frac{\left(E^{2}-m_{4}^{2} c^{4}\right)}{\hbar^{2} c^{2}} u_{r},
\end{gathered}
$$

\footnotetext{
${ }^{12}$ The situation is similar to the problem of a better description of hydrogen states by the Schrödinger equation than by the Klein-Gordon one.
} 
where

$$
\begin{aligned}
& U_{\text {Rep }}=\frac{L(L+1)+\left(\frac{m c A}{\hbar}\right)^{2}+3 \lambda_{d}\left(\frac{A}{d}\right)^{2}}{r^{2}} \equiv \frac{\mathcal{J}^{(2)}}{r^{2}}, \\
& U_{A t t r}=\frac{1}{A} \frac{\left(\left(\frac{m c A}{\hbar}\right)^{2}+\lambda_{d}\left(\frac{A}{d}\right)^{2}\right)}{r} \equiv \frac{\left(m_{4}^{2} c^{4}\right)}{(\hbar c)^{2}} \frac{A}{r},
\end{aligned}
$$

and

$$
\begin{aligned}
\mathcal{J}^{(2)} & =L(L+1)+\left(\frac{m c A}{\hbar}\right)^{2}+3 \lambda_{d}\left(\frac{A}{d}\right)^{2} \\
m_{4}^{2} c^{4} & =m^{2} c^{4}+\lambda_{d}\left(\frac{\hbar c}{d}\right)^{2} .
\end{aligned}
$$

Here $\mathcal{J}^{(2)}$ can be interpreted as the square of the total angular momentum and $m_{4}^{2}$ as the four-dimensional squared mass of the scalar field $\phi$ in the flat Minkowskian limit (see $[50,79])$. If the six-dimensional mass is zero $(m=0)$ then the four-dimensional mass at spatial infinity would be solely of kinematical origin. It is also easy to notice that neglecting the first term of (35) in the limit $r \rightarrow \infty$ is at this stage indistinguishable from zeroing the first derivative of the metric tensor ${ }^{13}$ in $\left(\frac{-r}{r+A}\right) \frac{\partial g^{r r}}{\partial r} \frac{\partial u_{r}}{\partial r}$ which has its origin in the first term at the LHS of (25).

Now, it is interesting to notice (see (38)) that the radial motion looks like the one dimensional motion of a particle with a unit mass in the potential $V_{\text {eff }}$

$$
V_{e f f}(r)=\frac{\hbar^{2}}{2}\left[U_{R e p}-U_{A t t r}\right]
$$

The first component on the right hand side can be interpreted as a modified centrifugal potential energy, and the second one as a potential energy related to some central attractive force. The existence of the second term is connected with the appearance of the effective coupling constant which is proportional to

$$
\epsilon=\frac{-\left(\frac{m c A}{\hbar}\right)^{2}-\lambda_{d}\left(\frac{A}{d}\right)^{2}}{A}=-\frac{m_{4}^{2} c^{2}}{\hbar^{2}} A .
$$

The value of $\epsilon$ depends both on the parameters and the quantum numbers of the model. After introducing $\beta=\alpha r$ we see [78] that (38) can be rewritten in the following form:

$$
\frac{1}{\beta^{2}} \frac{\partial}{\partial \beta}\left(\beta^{2} \frac{\partial u_{r}}{\partial \beta}\right)+\left[\frac{\iota}{\beta}-\frac{1}{4}-\frac{\mathcal{J}^{(2)}}{\beta^{2}}\right] u_{r}=0,
$$

where

$$
\begin{gathered}
\alpha^{2}=\frac{4\left(m_{4}^{2} c^{4}-E^{2}\right)}{\hbar^{2} c^{2}}, \\
\iota=\frac{-\epsilon}{\alpha},
\end{gathered}
$$

with $\mathcal{J}^{2}$ given by (41).

\footnotetext{
${ }^{13}$ Performing physics in $r \rightarrow \infty$ also means that we neglect in (35) a "radial force" proportional to $\partial u_{r} / \partial r$.
} 
Equation (45) has nearly the same formal shape as the radial equation obtained in the Schrödinger's model of the atom [78]. Now, converting (46) we obtain

$$
E^{2}=m_{4}^{2} c^{4}-\frac{\hbar^{2} c^{2} \alpha^{2}}{4}
$$

From the analysis of (45) it can be shown that the finite solutions for $\beta=0$ and $\beta \rightarrow \infty$ exist only when

$$
\iota=n^{\prime}+j+1,
$$

where $n^{\prime}$ is zero or a natural number and $j$ is a nonnegative solution of the equation

$$
j(j+1)=\mathcal{J}^{(2)}
$$

which is equal to

$$
j=-\frac{1}{2}+\frac{1}{2}\left[1+4 \mathcal{J}^{(2)}\right]^{\frac{1}{2}} .
$$

Finally, using (47) we can eliminate $\alpha$ from (48) and rewrite it in the following form:

$$
E^{2}=m_{4}^{2} c^{4}-\frac{1}{4} \hbar^{2} c^{2} \frac{(-\epsilon)^{2}}{\iota^{2}}=m_{4}^{2} c^{4}\left[1-\frac{1}{4} \frac{(-\epsilon)}{\iota^{2}} A\right],
$$

where

$$
\iota=n^{\prime}+\frac{1}{2}+\left[\frac{1}{4}+\mathcal{J}^{(2)}\right]^{\frac{1}{2}}
$$

and in the last equality in (52) the expression for $\epsilon$ given by (44) has been used.

At the end of this section let us notice that the original quantum Klein-Gordon equation given by (22) possesses the property of invariance according to which only the ratio $\frac{r}{A}$ matters. We will discuss the relevant symmetry below. Using this scale invariance, we could move from the microscopic to the astrophysical scale. In this context the calculations point to the similarity of both the 'neutron' and a galaxy dark matter structure (see also [50, 79]).

\subsection{Scale Invariance}

At this point the question concerning the value/s of $A$ arises. Previously [34-36] we considered the model given by (2) which has the following invariance:

$$
A \rightarrow \omega A, \quad r \rightarrow \omega r, \quad d \rightarrow \omega d, \quad c t \rightarrow \omega c t,
$$

where $\omega$ is the parameter of the transformation. This would mean that it is not the change of $A$ but rather of $\frac{A}{r}$ that matters. This is the invariance of the coupled Einstein (3) and KleinGordon (5) equations but it is not the invariance of their solution given by (13) and (16). This conclusion can also be drawn from (14). Hence we notice that the massless dilatonic field $\varphi$ is the Goldstone field [76]. What is more, using (11) and (14) in (15) we obtain

$$
R_{r}^{r}=-\kappa_{6}\left(\partial_{r} \varphi\right)^{2} g^{r r}=\frac{A^{2}}{2 r^{3}(r+A)} .
$$


We notice a similarity between this equation and its electromagnetic analog. ${ }^{14}$ Equation (55) is the (anti)screening current condition in gravitation, analogous to the screening current condition found in electromagnetism or in the electroweak sector in the self-consistent approach [36, 49].

On the level of the equations of motion (3) and (5) the theory is scale invariant unless mass $m$ enters. It would seem that invariance (54) leads to the invariance

$$
A \rightarrow \omega A, \quad r \rightarrow \omega r, \quad d \rightarrow \omega d, \quad c t \rightarrow \omega c t, \quad m \rightarrow \frac{1}{\omega} m
$$

of the Klein-Gordon equation (22). Yet the inclusion of any term with mass $m$ into the model given by (2) will inevitably lead to the gravitational coupling of the new matter with the metric tensor part of the Lagrangian, causing the breaking of the original invariance (54).

Hence the conclusion emerges that there appears a relation between the four-dimensional mass $m_{4}$ and the parameter $A$. It results from the Einstein equations which fix the value of $A$ after the self-consistent inclusion of the field $\phi$ with mass $m$ into the fields configuration. This means that the symmetry connected with the scaling (54) of $A$ is broken. Hence in (41) the dependance of $\mathcal{J}^{2}$ on $A$ for given $m_{4}$ is established, where $A$ is not a free parameter. In view of the lack of a full (self-consistent) analysis, $A$ should be estimated somehow and as we shall see this will appear possible in the weak limit approximation.

\subsection{Wave Mechanical Limit}

As we have proven that the global solution with $\lambda_{d}=0$ and $m=0$ does not exist (Sect. 3.1) hence its weak limit does not exist also. Therefore from now on we will investigate the cases with $\lambda_{d}>0$ only. The reason is that, as it has been discussed in Sect. 3.1, they only slightly perturb the metric-dilaton configuration given by (13) and (16). At the moment let us also suppose that $m=0$, a value which will be dynamically chosen in the next section.

We began with the Klein-Gordon equation (21) in which $\phi$ is the wave function, which in quantum mechanics is usually identified as the cause of angular momentum generators commutation relations. ${ }^{15}$ In our model the square of the total angular momentum $\mathcal{J}^{2}=$ $j(j+1)$ appears according to $(41)$ as the combination of terms $3 \lambda_{d}\left(\frac{A}{d}\right)^{2}$ and $L(L+1)$ which originate from the internal two-dimensional and orbital three-dimensional angular momenta, respectively. Yet, in the weak approximation regime these are $j$ and $L$ which take quantum mechanical values, therefore this means that $3 \lambda_{d}\left(\frac{A}{d}\right)^{2}$ chooses quantum mechanical values as a result of the proper addition of $j$ and $L$ only. Furthermore, since $j, L$ and $\lambda_{d}$ have quantum values hence $\frac{A}{d}$ also has this kind of values. The strange thing is that term $\frac{A}{d}$ appears as a result of the pure (field theory) classical interaction witch originates in the background metric and now has its part in $\mathcal{J}^{2}$. Next, as $\mathcal{J}$ manifests itself on the arena of the tree-dimensional space as the total angular momentum, we impose on it the usual quantum mechanical rules which lead to different representations for different values of $\mathcal{J}^{2}$.

This leads us to the indication of the place of wave mechanics (quantum mechanics) which from the point of view of the self-consistent field theory is discussed in [48, 49]. Wave mechanics appears as a theory for building a model of a system (e.g. an atomic one) with the lost of self-consistency, yet it partly works by describing its main characteristics.

\footnotetext{
${ }^{14}$ That is $\nabla^{2} \mathbf{A}=m_{A}^{2} \mathbf{A}$, where $\mathbf{A}$ is the electromagnetic vector potential.

${ }^{15}$ Yet they may be obtained from the commutation relations of rotation operations even before the quantum mechanics rules enter [80].
} 
By "partly" I mean that to receive a correction to the wave mechanics prediction the selfconsistency has to be reestablished. Yet this is the realm of quantum field theory or the self-consistent field theory [37-49, 81, 82]. To obtain both the main characteristics and the corrections the gravitational interactions should also be included in a self-consistent manner where the linear part of field fluctuations would be described by the quantum field theory. For the detailed discussion of the place of the quantum field theory see [37-47].

Below two examples for the presented 'gravitational Klein-Gordon' neutral particlelike solution in the weak interaction limit are given. The first one for a solution with the mass of $940 \mathrm{MeV}$, which could be called a 'neutron', and the second one with the mass of $1455 \mathrm{MeV}$ for a neutral meson named ' $\rho_{o}$ ' (presented briefly).

\section{Numerical Example: A 'Neutron' and Its Excited States}

Although the (internal) spin wave function could not have the $(\Theta, \Phi)$ space representation for the physical (half)-integer spin state, yet it does not eliminate motion as the origin of the spin in some kind of the classical space at all. Besides unknown, mainly three possibilities could be taken into account. The first one states that the parameters of the spin representations are "seen" in the interactions only without further inner explanation. The second one exists by extending the space by the Grassmann coordinates [83]. The third one, considered in this paper, constructs the spin of the particle from the motion in the geometry of the parametric space. The (half)-integer spin is then, as any other, just the numerical result.

The other spin representations of fields could have the similar origin (see Sect. 1.1). Yet there is a reason to consider them as having the different dimensions of their parametric spaces. The proof goes via the Fisherian statistical analysis performed by Frieden [62-70] and the conclusion is that the dimension of the parametric space depends on the species of the field of the rank $\aleph$ which is the dimension of the sample collected by the field alone during its sampling of the space-time. For the complex scalar field the dimension of the extra parametric space is equal to $\aleph=2$. For the other fields this dimension is bigger, e.g $\aleph=4$ for the electromagnetic field and $\aleph=8$ for the Dirac one. Hence, as it has been mentioned previously, the four-dimensional space-time could be extended simultaneously to the variety of the multi-dimensional ones.

\section{1 'Neutron'}

Let us make some numerical adjustments for the parameters of the presented model $(\aleph=2)$. For this purpose we begin with the state which possesses the following value of the internal quantum number (see Sects. 3.1-3.2):

$$
\lambda_{d}=1
$$

choosing the following value of the four dimensional mass:

$$
m_{4} \approx 952.893194 \mathrm{MeV} / \mathrm{c}^{2},
$$

which is a little bit bigger than the observed mass of the neutron $939.56536 \mathrm{MeV} / \mathrm{c}^{2}$. Solving (42) we obtain

$$
d=d_{l} \approx 2.071 \cdot 10^{-16} \mathrm{~m}=0.2071 \mathrm{fm}
$$


as the limit value, i.e. from (42) we see that for $d>d_{l}$ we obtain $m^{2}>0$ and for $d<d_{l}$ we obtain $m^{2}<0$ which is a tachion. For the limit value $m^{2}=0$ the second term of $\mathcal{J}^{2}$ at the RHS of (41) is equal to

$$
\left(\frac{m c A}{\hbar}\right)^{2}=0
$$

Now, it is interesting to notice that the RHS of (41) might be interpreted as $j(j+1)$ (see (50)), which means that

$$
\begin{aligned}
\mathcal{J}^{(2)} & =L(L+1)+\left(\frac{m c A}{\hbar}\right)^{2}+3 \lambda_{d}\left(\frac{A}{d}\right)^{2} \\
& =j(j+1) .
\end{aligned}
$$

After taking $L=0$ and $j=1 / 2$ for the 'neutron' the equation on the relative value of $A$ and $d$ is obtained from the above equation. Moreover, we see that (61) gives us

$$
\frac{A}{d}=1 / 2 \text { for } d=d_{l}\left(m^{2}=0\right) .
$$

Now, according to (52) and (44) we obtain for $m=0$ and $\frac{A}{d}$ as in (62) the following equation

$$
E^{2}=m_{4}^{2} c^{4}\left[1-\frac{1}{4} \frac{(-\epsilon A)}{\iota^{2}}\right]=m_{4}^{2} c^{4}\left[1-\frac{1}{36}\left(\frac{3 / 2}{\iota}\right)^{2}\right]
$$

and using in this equation $m_{4}$ from (58) we find that the energy of the 'neutron' in the ground state $\left(n^{\prime}=0, j=1 / 2\right.$ and $\iota=3 / 2$, see (49)) is equal to

$$
E=939.565 \mathrm{MeV} / \mathrm{c}^{2}
$$

which is the observed mass of the neutron. From the second term of (63) it results that the gravitational correction to the energy of the 'nucleon' in the ground state $\left(n^{\prime}=0\right.$ and $j=1 / 2$ and $\iota=3 / 2$ ), i.e. the gravitational bounding energy, is equal to

$$
\Delta E=E-m_{4} c^{2} \approx-0.0139867 m_{4} c^{2} .
$$

Finally, let us recall that the solution for $\lambda_{d}=1$ has according to (33) and (34) the double degeneracy: ( $l=0$ and $\tilde{\mathrm{m}}=1$-which we interpret as the spin up solution) or $(l=0$ and $\tilde{\mathrm{m}}=-1-$ which we interpret as the spin down solution). This assignment is very natural but by its own it does not lead to the conclusion that the particle is the neutron. The calculation of the energy of the ground state of a field having the "quantum" numbers characteristic for the neutron entitles only to the conclusion that the formalism could describe the neutron. Hence we see that the model points to the origin of the spin of the nucleon as connected with the geometry of the internal manifold and with motion inside its two compactified parametric dimensions. Moreover, it indicates that gravitational effects give a visible change of the spectrum of the levels in the proposed model of the nucleon.

As it has been shown, the background gravity-dilatonic configuration given by (13) and (16) is stable (see Sect. 2.1). Also the ground state solution obtained in Sect. 3 for the scalar field $\phi$ is the stationary one. Moreover, it perturbs the background configuration slightly only (see Sect. 3.1) and in the discussed weak approximation we could argue that the 
whole configuration of the fields, $g_{M N}, \varphi$ and $\phi$, is stable. Finally, for a particle with another value of its spin the dimension $\aleph$ of the parametric space is different, yet the conclusion, if the relevant calculations are performed, would be similar.

\subsection{The Value of $m=0$}

Let us still consider one particular state (e.g. neutron) with $m_{4}^{2}$ fixed, i.e. $m$ and $d$ may vary leaving $m_{4}^{2}$ unchanged. Now, with the use of (42) we notice that (61) can be rewritten as follows

$$
\begin{aligned}
\mathcal{J}^{(2)} & =j(j+1) \\
& =L(L+1)+m_{4}^{2} c^{4}\left(\frac{A}{c \hbar}\right)^{2}+2 \lambda_{d}\left(\frac{A}{d}\right)^{2}
\end{aligned}
$$

hence we see that $\mathcal{J}^{2}$ for $\lambda_{d}>0$ is not proportional to $m_{4}^{2} c^{4}$ (no matter what the integer $L$ is) and it is worth noting that to keep $\mathcal{J}^{2}$ equal to $j(j+1)$ for $j$ equal to the multiplicity of $1 / 2$, it is necessary to have $m=0$ (hence $m_{4}^{2} c^{4}=\lambda_{d}\left(\frac{\hbar c}{d}\right)^{2}$ ) without any variation from this particular value of $m$.

\subsection{Excited States}

As in Sect. 4.1 the value of $m_{4}$ for the 'neutron' has been chosen to fit the value of the observed energy $E$ of the neutron hence it is important to calculate the predictions for values of the energy of its excited states. Let us make the following assumption on the relation between the total angular momentum number and the orbital one:

$$
j=L+\frac{1}{2}
$$

For the mass $m=0$ we may notice from (61) that the characteristic radius $A$ has to change with $j, L$ and $\lambda_{d}$ in the following way

$$
A=\frac{d \sqrt{j(1+j)-L(1+L)}}{\sqrt{3} \sqrt{\lambda_{d}}} \quad \text { for } m=0,
$$

where $d$ is kept equal to $d_{l}=0.2071[\mathrm{fm}]$ as in (59). Then, with $m_{4}$ given for each particular state by (42), the energy $E$ is calculated according to (52). The numerical values of the energy $E$ for the ground state $\left(j=\frac{1}{2}, \lambda_{d}=1\right)$ and excited states of the 'neutron' are given in the Table 1 below.

Except for the ground state (which has been chosen to fit the neutron observed energy) all other energies are equal to the observed ones approximately only [84-88]. Nevertheless this might not be a big shortcoming of the model since we use the weak approximation only. This statement appears to be sensible, especially in the light of the agreement of the order of the discrepancies between energies of the states belonging to the same column and different rows, which are of the order found in the experiment. As the spacial configurations for the first column in the Table 1 are the most simple ones $(L=0)$ hence the feeling is that only they should lie in the neighborhoods of the true states (see also the discussion after (35)). 
Table 1 Values of energies $E \mathrm{MeV}$ for the ground state and excited states of the 'neutron' as the function of the total angular momentum number $j$ and internal quantum number $\lambda_{d}$. For the 'neutron' itself $j=\frac{1}{2}$ and $\lambda_{d}=1$. The last two columns present the gravitational bounding energy $\Delta E \mathrm{MeV}$ and the ratio $A / d$ for the 'neutron' and its excited states with $j=1 / 2$ and $L=0$. All states in Table 1 have $m=0$ and $d=d_{l}=0.2071$ fm (see the text)

\begin{tabular}{lccccll}
\hline$\lambda_{d}$ & $j=1 / 2$ & \multicolumn{1}{c}{$3 / 2$} & $5 / 2$ & $7 / 2$ & $\Delta E\left(j=\frac{1}{2}\right)$ & $A / d\left(j=\frac{1}{2}\right)$ \\
\hline 1 & $\mathbf{9 3 9 . 5 6 5}$ & 941.71 & 943.938 & 945.512 & $\mathbf{- 1 3 . 3 2 7 8}$ & $\mathbf{0 . 5}$ \\
2 & $\mathbf{1 3 2 8 . 7 5}$ & 1331.78 & 1334.93 & 1337.16 & $\mathbf{- 1 8 . 8 4 8 4}$ & $\mathbf{0 . 3 5 3 6}$ \\
3 & $\mathbf{1 6 2 7 . 3 7}$ & 1631.09 & 1634.95 & 1637.67 & $\mathbf{- 2 3 . 0 8 4 5}$ & $\mathbf{0 . 2 8 8 7}$ \\
4 & $\mathbf{1 8 7 9 . 1 3}$ & 1883.42 & 1887.88 & 1891.02 & $\mathbf{- 2 6 . 6 5 5 7}$ & $\mathbf{0 . 2 5}$ \\
6 & $\mathbf{2 3 0 1 . 4 6}$ & 2306.71 & 2312.17 & 2316.02 & $\mathbf{- 3 2 . 6 4 6 4}$ & $\mathbf{0 . 2 0 4 1}$ \\
& $L=0$ & 1 & 2 & 3 & $L=0$ & $L=0$ \\
\hline
\end{tabular}

\subsubsection{The Gravitational Bounding Energy}

Additionally, in the two last columns of the Table 1, the values of the gravitational bounding energy

$$
\Delta E=E-m_{4} c^{2}=m_{4} c^{2}\left[\sqrt{1-\frac{1}{4} \frac{(-\epsilon A)}{\iota^{2}}}-1\right],
$$

for the states with $j=\frac{1}{2}$ and $L=0$ are given. Here, according to (44) and (42) the effective coupling constant $\epsilon$ and the four-dimensional squared mass $m_{4}^{2}$ are for $m=0$ equal to

$$
\epsilon=-\frac{m_{4}^{2} c^{2}}{\hbar^{2}} A \quad \text { and } \quad m_{4}^{2} c^{4}=\lambda_{d}\left(\frac{\hbar c}{d}\right)^{2} \quad \text { for } m=0,
$$

where $A$ is given by (68).

The important result is that for fixed $j$ and $L$, the increase of the energy $E$ of the configuration causes the system to become more tightened (as the ratio $A / d$ decreases) and simultaneously its gravitational bounding energy bigger. As an example, for the 'neutron' and its excited states (with $j=1 / 2$ and $L=0$ ) this result has been presented in the first and in the two last columns of the Table 1 (in bold).

The model could be applied to the other known particles also, yet the present calculations show that the best agreement is achieved for the neutron and its excited states. For example, for the neutral meson ' $\rho_{o}$ ' taken as the ground state (for $\rho(1450)$ inside $1465 \pm 25 \mathrm{MeV}$ ) and for its first five excited states (for all of them $j=1, n^{\prime}=0, \iota=2$ and $L=0$ ), we obtain the energies equal to $1454.5,2056.97,2519.26,2909.0,3562.78,3848.24 \mathrm{MeV}$ for $\lambda_{d}$ equal to $1,2,3,4,6,7$, respectively. In this case the matching with the experimental values [84-88] is worse than in the case of the neutron. The explanation of this fact might be that the gravitational factor in the structure of the neutron, which is the lowest known barionic neutral ground state, is more prominent than in the case of other neutral barions.

\subsubsection{The Problem with the Degeneracy for $\lambda_{d}$}

The principal problem seems to be connected with the ladder of the energy state values in each particular row since in each row a few or may be only one are physical. This problem 
might be connected with the zeroing of the term with the first derivative of the metric tensor and hence with the removal of the first derivative of the radial wave function $u_{r}$ on passing from (35) to (36). Unfortunately, after this zeroing, the weak limit approximation was applied. This leads effectively to the introduction of an extra force term (equal to the neglected one with the opposite sign) connected with the mathematics of the weak approximation only. The addition of this unphysical extra force into the system results in the appearance of row ladders of the unphysical states. Yet the unphysical differences between states of the ladder in one particular row with the established value of $\lambda_{d}$ are two order of magnitude smaller than the physical ones from the columns. This agrees with the fact that the differences in one row appear as the result of the change in the value of $L$. Now, according to Sect. 3.3 the orbital angular momentum $L$ remained with the third term $\left(\frac{r}{r+A}\right) \frac{L(L+1)}{r^{2}} u_{r}$ of (35). Yet it remained inconsistently since the first term $\frac{A}{r^{2}}\left(\frac{r}{r+A}\right) \frac{\partial u_{r}}{\partial r}$ of this equation, also proportional to $\frac{1}{r^{2}}$, has been neglected. Since the third term of (35) is, for $r \rightarrow \infty$, of an order smaller than the ones which remained together with it in (36) (and consequently in (38)) then the energy differences in a particular row are also smaller than in a particular column. The analysis of (38)-(40) is in agreement with the remarks above, i.e. the change of $L$ in $U_{R e p} \sim \frac{1}{r^{2}}$ is connected with the small energy differences in a particular row whereas the change of $\lambda_{d}$ in $U_{\text {Attr }} \sim \frac{1}{r}$ is connected with big energy differences in a particular column in Table 1 . In Sect. 3.3 the term with $L$ has been left for the sake of the angular momentum considerations.

\section{Conclusions}

Till now there is no established experimental evidence for multi-dimensionality of the world or it is possible that our understanding of potential manifestations of higher dimensions is still poor. Yet at the same time there exist noticeable literature references indicating that the pursued effects of extra dimensions could have already been noticed earlier both in the astrophysical [34-36, 50, 79, 89-97] and in the elementary particle scale [48, 98-104]. These findings are in contradistinction to the standard expectations that extremely high energies are necessary to probe higher dimensions. The renewed interest in the KaluzaKlein theories, to which the presented six-dimensional model belongs, stems from the fact that multidimensional analogues of general relativity are able, among others, to generate the four dimensional mass. This would be the outcome of the interaction which proceeds both from the four-dimensional world and the internal space dimensions (here two parametric ones) with currents of the matter which modify masses in the four-dimensional world (see also [50]). Our approach to the modelling of a system, e.g. of one particle (here neutron), reflects the hope that its structure can be described by the dynamics in the six-dimensional space-time. Especially the characteristics of the structure such as its spin and, at least partly, its inertia have their origin in the internal two-dimensional parametric space. This is the reason for perceiving multi-dimensional (here six-dimensional $)^{16}$ theories as still attractive for understanding the fundamental interactions.

To fulfil this aim the present paper examines the implementation of the geometrical properties of the previously [34, 35] worked out six-dimensional Kaluza-Klein type model. These properties may manifest themselves in the realm of particle physics, e.g. by affecting the structure of the neutron and its excited states. The obtained ground state solution appeared to have two spinorial degrees of freedom since the total angular momentum with $j=1 / 2$

\footnotetext{
${ }^{16}$ For example, in the case of electromagnetism for which $\aleph=4$, the space-time would be eight-dimensional.
} 
goes (for the main internal angular quantum number $\lambda_{d}=1$ ) with two internal magnetic quantum number possibilities, $\tilde{\mathrm{m}}=1$ or $\tilde{\mathrm{m}}=-1$.

In Sect. 1.1, the statistical Fisherian reasons for the six-dimensionality of the space-time are given. The basics of the model are presented in Sect. 2. We recall here the static spherically symmetric "reference" back-ground solution of the six-dimensional Einstein equations coupled with the Klein-Gordon equation, where the dilatonic field is the basic one and the gravitational field is the self-field [34, 35]. A more detailed discussion of its properties is presented in [34-36, 50, 79]. The metric tensor part of the "reference" solution seems analogous to the familiar four-dimensional Schwarzschild solution, however it differs fundamentally since it is horizon free. In Sect. 2.1 the stability of the "reference" self-consistent solution is shown. Section 3 is devoted to the derivation of the weak limit approximation of the general wave mechanical (quantum mechanical) approach defined in the context of physical modelling which loses its self-consistency (here the gravitational one). Hence a new scalar field has been added to the original "reference" configuration of fields and the non self-consistent case for its Klein-Gordon equation is defined. Here the main purpose is to find the solution of this Klein-Gordon equation and the reason for doing this is twofold. Firstly it is connected with the derivation of the energy of states and secondly with the indication of the spin origin of the configuration as the manifestation of both the geometry of the internal two-dimensional parametric space and of the kinematics of the fields inside it. In Sect. 3.1 the problem of the departure from the (gravitational) self-consistent calculations of the metric and other fields of the configuration is discussed. The loss of self-consistency is due to the neglect of the total energy-momentum tensor perturbation term, which is proportional to $\hbar^{2}$. In Sect. 4 the implementation of the model to the description of the 'neutron' and its excited states is performed together with the derivation of their energy. Finally, in Sect. 4.3 the discussion of the consequences of the zeroing of the first derivative term in the radial Klein-Gordon equation is given.

The question arises on the status of the presented model of the neutron. As discussed in Sects. 3 and 4 it is interpreted as the wave mechanical departure from the fully (i.e. including gravitation) self-consistent field theory understood in accordance with Schrödinger's substantial interpretation of the wave function $[48,49]$. The wave mechanical approach means that the self-consistency is lost. However with any hope a fully self-consistent model incorporating all the necessary fields and interactions will result not only in the refinement of the proposed model of the neutron and its excited states but also in the construction of better models for all other elementary matter particles. Yet this is a difficult task to perform [37-49, 81, 82]. In today's physics mainly the wave mechanical (weak approximation case) and quantum field theory (linear) regimes are noticed. Yet self-consistent exceptions are also quoted, developed only for the description of the linear fluctuations of the matter wave function [37-47]. The concept which lies behind this approach is not the one of the quantum theory of interactions, with quantum gravity included [105], where the second quantization procedure for a set of aggregated quanta is realized. But, it is rather the concept of a relativistic self-consistent field theory which takes into account all interactions including gravity. The theoretical basis which is stronger than the mean-field theory concept guarantees the consistency of this approach since both wave mechanics and classical field theories can be understood as having the same, Fisher-information origin with finite $\aleph$ [62-73].

Finally, in this paper the mass of a particle originates from both the external and the internal dimensions, where the internal part of the mass is of purely kinetic origin. The final mass of the particle may be calculated in accordance with a broadened version of Thrirring's and Einstein's analysis of Einstein's geometrodynamics or in accordance with an effective gravity theory of the Logunov type [75]. In both cases the internal contribution to the mass 
should appear as the result of self-consistent calculations. Interestingly the same conclusions were drawn by Frieden and Soffer [62-71] from the statistical method of estimating the physical models. It has recently been proven in [72, 73], where the entanglement between the kinematical and structural degrees of freedom has been rigorously established. Now from our considerations it results that since the mass has its origin in the dynamics and in the kinetic motion of the (extended) field of every elementary particle inside the parametric space, then we can conclude that every particle is a continuously extended object.

Yet, the presented model is obviously not the final one. The reason is twofold. At first, from its beginning it was formulated for the neutral particle only. The inclusion, for instance, of the electric or effective weak charges, which would be necessary, changes the results. ${ }^{17}$ Secondly, the first particle in every chosen ladder of states should really be the ground state of the configuration of all fields, which are introduced to describe self-consistently both the ground state and the excited ones. Hence, further calculations should take into account more realistic shapes of the main charge densities incorporating their fluctuations for the extended basic matter sources to which proper self-fields are coupled. These shapes should follow, for instance, from the Einstein equations coupled to Klein-Gordon-Maxwell's ${ }^{18}$ or Dirac-Maxwell's set of equations for charge densities with fluctuations included, as required for self-consistent models [49]. This means that from the mathematical point of view, a matter particle seems to be a self-consistent solution of field equations for the basic fields including fluctuations and their self-fields. These self-fields have to be interwoven in the description of the matter particle. The presented model indicates the point where the weak, wave mechanical limit of the theory should be placed. It is also a step towards the construction of a self-consistent formalism of the classical theory for a single, continuously extended, elementary particle. The final model, however, still belongs to the realm of the unknown.

Acknowledgements This work has been supported by L.J.Ch.. Special thanks to Barbara Mierzyńska for the correction of the text. This work has been also supported by the Department of Field Theory and Particle Physics, Institute of Physics, University of Silesia, the project "Teoretyczne i fenomenologiczne badanie Modelu Standardowego i jego uogólnieñ", No.: BS-03-0507-022-07 and by the Junta de Andalucía project FQM 437. I would also like to extend my thanks to Heptools for its support.

Open Access This article is distributed under the terms of the Creative Commons Attribution Noncommercial License which permits any noncommercial use, distribution, and reproduction in any medium, provided the original author(s) and source are credited.

\section{Appendix A: The Components of the Ricci Tensor of the Background Metric}

Using (7)-(8), we can calculate the components of the Ricci tensor. The nonvanishing components are [34-36]

$$
\begin{aligned}
R_{t}^{t}= & \left(4 \varrho^{2} r v^{\prime}+4 r^{2} \varrho^{\prime} \varrho v^{\prime}-r^{2} \varrho^{2} \mu^{\prime} v^{\prime}+r^{2} \varrho^{2}\left(v^{\prime}\right)^{2}\right. \\
& \left.+2 r^{2} \varrho^{2} v^{\prime \prime}\right)\left(4 e^{\mu} r^{2} \varrho^{2}\right)^{-1}
\end{aligned}
$$

\footnotetext{
${ }^{17}$ For the self-consistent analysis in the classical counterpart of the electroweak model see [49].

${ }^{18}$ Instead of Maxwell equations, the Yang-Mills ones are sometimes taken into considerations. However it is probable that in future theories they will be replaced by the still unknown equations.
} 


$$
\begin{aligned}
R_{r}^{r}= & \left(-4 \varrho^{2} r \mu^{\prime}-4 r^{2} \varrho^{\prime} \varrho \mu^{\prime}-r^{2} \varrho^{2} \mu^{\prime} v^{\prime}+r^{2} \varrho^{2}\left(v^{\prime}\right)^{2}\right. \\
& \left.+8 r^{2} \varrho \varrho^{\prime \prime}+2 r^{2} \varrho^{2} v^{\prime \prime}\right)\left(4 e^{\mu} r^{2} \varrho^{2}\right)^{-1} \\
R_{\Theta}^{\Theta}= & R_{\Phi}^{\Phi}=\left(-4 e^{\mu} \varrho^{2}+4 \varrho^{2}+8 r \varrho \varrho^{\prime}-2 r \varrho^{2} \mu^{\prime}\right. \\
& \left.+2 r \varrho^{2} v^{\prime}\right)\left(4 e^{\mu} r^{2} \varrho^{2}\right)^{-1} \\
R_{\vartheta}^{\vartheta}= & R_{\varsigma}^{\varsigma}=\left(8 \varrho r \varrho^{\prime}+4 r^{2}\left(\varrho^{\prime}\right)^{2}-2 r^{2} \varrho \varrho^{\prime} \mu^{\prime}\right. \\
& \left.+2 r^{2} \varrho \varrho^{\prime} v^{\prime}+4 r^{2} \varrho \varrho^{\prime \prime}\right)\left(4 e^{\mu} r^{2} \varrho^{2}\right)^{-1}
\end{aligned}
$$

\section{Appendix B: The Weak Interaction Limit Coefficients}

The coefficients of expansions of the radial equation (37) and their limits for $x_{0}=0$ $(r \longrightarrow \infty)$ are equal to

$$
\begin{array}{ll}
p_{0} & =-\frac{x_{0}^{3}}{\left(1+x_{0}\right)^{3}}=0, \quad p_{1}=\frac{3 x_{0}^{2}+x_{0}^{3}}{\left(1+x_{0}\right)^{3}}=0, \\
p_{2} & =\frac{1}{\left(1+x_{0}\right)^{3}}=1, \\
\mu_{0} & =\frac{1+3 x_{0}+3 x_{0}^{2}}{\left(1+x_{0}\right)^{3}}=1, \quad \mu_{1}=-\frac{1+3 x_{0}}{\left(1+x_{0}\right)^{3}}=-1, \\
\mu_{2} & =\frac{1}{\left(1+x_{0}\right)^{3}}=1, \\
\lambda_{0} & =\frac{1+3 x_{0}+5 x_{0}^{2}}{\left(1+x_{0}\right)^{4}}=1, \\
\lambda_{2} & =\frac{3}{\left(1+x_{0}\right)^{4}}=3 .
\end{array}
$$

\section{References}

1. Kelvin, L.: Aepinus revisited. Philos. Mag. VI 3, 257-283 (1902)

2. Kelvin, L.: On the weights of atoms. Philos. Mag. VI 4, 177-198 (1902)

3. Thomson, J.J.: Philos. Mag. 7, 237 (1904)

4. Chemical Rubber Co.: Handbook of Chemistry and Physics, 68th edn., p. F-157. CRC, Boca Raton (1987)

5. Maxwell, J.C.: On theories of the constitution of Saturn's rings. Edinb. R. Soc. Proc. IV, 99-101 (1862)

6. Thomson, J.J.: Cathode rays. Philos. Mag. 44, 293-316 (1897)

7. Stoney, G.J.: On the 'electron' or atom of electricity. Lett. Philos. Mag. 38, 418-420 (1894)

8. De Luca, J., Rodrigues, S.B., Levin, Y.: Electromagnetic instability of the Thomson problem. Europhys. Lett. 71, 84 (2005). cond-mat/0506616

9. Rutherford, E.: Proc. R. Soc. A 97, 394 (1920)

10. Heisenberg, W.: Über quantenmechanische Umdeutung kinematischer und mechanischer Beziehungen. Z. Phys. 33, 879-893 (1925)

11. Dirac, P.A.M.: The fundamental equations of quantum mechanics. Proc. R. Soc. Lond., Ser. A 109, 642-653 (1925)

12. Dirac, P.A.M.: Quantum mechanics and a preliminary investigation of the hydrogen atom. Proc. R. Soc. Lond., Ser. A 110, 561-579 (1926)

13. Dirac, P.A.M.: The quantum theory of the electron. Proc. R. Soc. Lond., Ser. A 117, 610-624 (1928) 
14. Dirac, P.A.M.: The quantum theory of the electron. Proc. R. Soc. Lond., Ser. A 118, 351-361 (1928)

15. Schrödinger, E.: Quantisierung als Eigenwertproblem. (Erste Mitteilung.) Ann. Phys. 79, 361-376 (1926)

16. Schrödinger, E.: Quantisierung als Eigenwertproblem. (Zweite Mitteilung.) Ann. Phys. 79, 489-527 (1926)

17. Schrödinger, E.: Quantisierung als Eigenwertproblem. (Dritte Mitteilung: Storungstheorie mit Anwendung auf den Starkeffekt der Balmerlinien). Ann. Phys. 80, 437-490 (1926)

18. Schrödinger, E.: Quantisierung als Eigenwertproblem. (Vierte Mitteilung.) Ann. Phys. 81, 109-139 (1926)

19. de Broglie, L.: Recherches sur la theorie des quanta. Ph.D. dissertation, Paris (1924)

20. Espinosa, J.M.: Physical properties of de Broglie's phase waves. Am. J. Phys. 50, 357-362 (1982)

21. Albert, A.A.: Trans. Am. Math. Soc. 64, 552 (1948)

22. Santilli, R.M.: Hadron. J. 1, 224 (1978)

23. Santilli, R.M.: Hadron. J. 1, 574 (1978). Also see p. 1267

24. Santilli, R.M.: Communication of the JINR, Dubna, Russia, $\sharp$ E4-93-352 (1993)

25. Santilli, R.M.: Chin. J. Syst. Ing. Electron. 6, 177 (1996)

26. Lie, S.: Over en classe geometriske transformationer. Algebras Groups Geom. 15, 395 (1998). English translation by E. Trell

27. Stiegler, U.: Phys. Rep. 277, 1 (1996)

28. Zheng, X., et al. (the Jefferson Lab Hall A Collaboration): Precision measurement of the neutron spin asymmetry $A_{1}^{n}$ and spin-flavor decomposition in the valence quark region. Phys. Rev. Lett. 92, 012004 (2004). nucl-ex/0308011

29. Heyde, K.: Basic Ideas and Concepts in Nuclear Physics, 3rd edn., p. 577. IOP Publishing, Bristol (2004)

30. Riemann, G.F.B.: Bei die Hypothesen, welche der Geometrie zur Grunde liegen. In: Gesammelte Mathematische Werke (1866). Reprint of 2nd edn., H. Weber (ed.), Dover, New York (1953). See also the translation by W.K. Clifford, Nature 8, 14-17 (1973)

31. Lakhtakia, A. (ed.): Models and Modelers of Hydrogen. Singapore, World Scientific (1996)

32. Sallhofer, H.: Hydrogen in electrodynamics. VI. The general solution. Z. Naturforsch. 45a, 1361-1366 (1990)

33. Sakurai, J.J.: Advanced Quantum Mechanics, p. 169. Addison-Wesley, Reading (1967)

34. Biesiada, M., Mańka, R., Syska, J.: A new static spherically symmetric solution in six-dimensional dilatonic Kaluza-Klein theory. Int. J. Modern Phys. D 9(1), 71-78 (2000)

35. Mańka, R., Syska, J.: Nonhomogeneous six-dimensional Kaluza-Klein compactification. Preprint UŚLTP-95/03, University of Silesia (1995)

36. Syska, J.: Self-consistent classical fields in gauge field theories. Ph.D. thesis (unpublished), University of Silesia $(1995 / 1999)$

37. Barut, B.O., Kraus, J.: Nonperturbative quantum electrodynamics: the Lamb shift. Found. Phys. 13, 189-194 (1983). doi:10.1007/BF01889480

38. Barut, A.O., Van Huele, J.F.: Quantum electrodynamics based on self-energy: Lamb shift and spontaneous emission without field quantization. Phys. Rev. A 32, 3187-3195 (1985). doi:10.1103/PhysRevA.32.3187

39. Barut, A.O., Dowling, J.P.: Quantum electrodynamics based on self-energy: spontaneous emission in cavities. Phys. Rev. A 36, 649-654 (1987). doi:10.1103/PhysRevA.36.649

40. Barut, A.O.: Combining relativity and quantum mechanics: Schrödinger's interpretation of $\psi$. Found. Phys. 18, 95-105 (1988). doi:10.1007/BF01882875

41. Barut, A.O.: Schrödinger's interpretation of psi as a continuous charge distribution. Ann. Phys. (Leipzig) 45, 31-36 (1988)

42. Barut, A.O.: The revival of Schrödinger's interpretation of quantum mechanics. Found. Phys. Lett. 1, 47-56 (1988). doi:10.1007/BF00661316

43. Barut, A.O.: Quantum-electrodynamics based on self-energy. Phys. Scr. T 21, 18-21 (1988)

44. Barut, A.O., Salamin, Y.I.: Relativistic theory of spontaneous emission. Phys. Rev. A 37, 2284-2296 (1988). doi:10.1103/PhysRevA.37.2284

45. Barut, A.O., Ünal, N.: An exactly soluble relativistic quantum two-fermion problem. J. Math. Phys. 27, 3055 (1986). doi:10.1063/1.527235

46. Barut, A.O., Ünal, N.: A new approach to bound-state quantum electrodynamics: I. Theory. Physica A 142, 467-487 (1987). doi:10.1016/0378-4371(87)90036-7

47. Barut, A.O., Ünal, N.: A new approach to bound-state quantum electrodynamics: II. Spectra of positronium, muonium and hydrogen. Physica A 142, 488-497 (1987). doi:10.1016/0378-4371(87)90037-9

48. Syska, J.: Remarks on self-consistent models of a particle. In: Ling, A.V. (ed.) Trends in Boson Research, pp. 163-181. Nova Science, New York (2006) 
49. Syska, J.: Boson ground state fields in the classical counterpart of the electroweak theory with non-zero charge densities. In: Kovras, O. (ed.) Frontiers in Field Theory, pp. 125-154. Nova Science, New York (2005), Chap. 6. Also in Focus on Boson Research, pp. 233-258. Nova Science, New York (2006)

50. Biesiada, M., Syska, J.: Motion of test particles in six-dimensional dilatonic Kaluza-Klein theory, in review (2010)

51. Nishino, M., Sezgin, E.: Phys. Lett. B 144, 187 (1984)

52. Salam, A., Sezgin, E.: Phys. Lett. B 147, 47 (1984)

53. Mańka, R., Syska, J.: Torus compactification of six-dimensional gauge theory. J. Phys. G: Nucl. Part. Phys. 15, 751-764 (1989). doi:10.1088/0954-3899/15/6/007

54. Ivashchuk, V.D., Melnikov, V.N.: Class. Quantum Gravity 11, 1793 (1994)

55. Ivashchuk, V.D., Melnikov, V.N.: Gravit. Cosmol. 1(2), 133 (1995)

56. Ivashchuk, V.D.: Ph.D. dissertation, VNICPV, Moscow (1989)

57. Bronnikov, K.A., Melnikov, V.N.: Ann. Phys. (N.Y.) 239, 40 (1995)

58. Sparling, G.A.J.: Proc. R. Soc. A 1839 (2007). doi:10.1098/rspa.2007

59. Demers, P.: Can. J. Phys. 43, 1687 (1975)

60. Cole, E.A.B.: Nuovo Cimento A 40, 171 (1977)

61. Patty, C.E. Jr., Smalley, L.L.: Phys. Rev. D 32(4), 891-897 (1985)

62. Frieden, B.R.: Found. Phys. 16, 883 (1986)

63. Frieden, B.R.: Fisher information, disorder, and the equilibrium distributions of physics. Phys. Rev. A 41, 4265 (1990). doi:10.1103/PhysRevA.41.4265

64. Frieden, B.R., Soffer, B.H.: Lagrangians of physics and the game of Fisher-information transfer. Phys. Rev. E 52(3), 2274-2286 (1995)

65. Frieden, B.R., Plastino, A.: Composite fermion particles and Fisher information. Phys. Lett. A 272, 326-332 (2000)

66. Frieden, B.R., Plastino, A.: Higgs mass generation from the standpoint of information. Phys. Lett. A 278, 299-306 (2001)

67. Frieden, B.R.: Relations between parameters of a decoherent system and Fisher information. Phys. Rev. A 66, 022107 (2002). doi:10.1103/PhysRevA.66.022107

68. Frieden, B.R., Plastino, A., Plastino, A.R., Soffer, B.H.: Schrödinger link between nonequilibrium thermodynamics and Fisher information. Phys. Rev. E 66, 046128 (2002). doi:10.1103/ PhysRevE.66.046128

69. Frieden, B.R., Plastino, A., Plastino, A.R., Soffer, B.H.: Phys. Lett. A 304, 73 (2002)

70. Frieden, B.R.: Science from Fisher Information: A Unification. Cambridge University Press, Cambridge (2004)

71. Frieden, B.R., Soffer, B.H.: Information-theoretic significance of the Wigner distribution. Phys. Rev. A 74, 052108 (2006). doi:10.1103/PhysRevA.74.052108

72. Syska, J.: Fisher information and quantum-classical field theory: classical statistics similarity. Phys. Status Solidi B 244(7), 2531-2537 (2007). doi:10.1002/pssb.200674646, arXiv:0811.3600

73. Piotrowski, E.W., Sładkowski, J., Syska, J., Zając, S.: The method of the likelihood and the Fisher information in the construction of physical models. Phys. Status Solidi B 246(5), 1033-1037 (2009). doi:10.1002/pssb.200881566, arXiv:0811.3554

74. Amari, S., Nagaoka, H.: Methods of Information Geometry. Oxford University Press, Oxford (2000), Chap. 2.6

75. Denisov, V.I., Logunov, A.A.: The theory of space-time and gravitation. In: Logunov, A.A. (ed.) Gravitation and Elementary Particle Physics. Physics Series, pp. 14-130. MIR, Moscow (1983)

76. Green, M.B., Schwarz, J.H., Witten, E.: Superstring Theory, vol. 2. Cambridge University Press, Cambridge (1988), Chap. 13

77. Chen, S., Wang, B., Su, R.: Phys. Lett. B 647, 282-287 (2007)

78. Schiff, L.I.: Quantum Mechanics, 3rd edn. (1968)

79. Biesiada, M., Rudnicki, K., Syska, J.: An alternative picture of the structure of galaxies. In: Rudnicki, K. (ed.) Gravitation, Electromagnetism and Cosmology: Toward a New Synthesis, p. 31. Apeiron, Montreal (2001)

80. Sakurai, J.J.: Modern Quantum Mechanics. Addison-Wesley, Reading (1994). San Fu Tuan (ed.)

81. Jaynes, E.T.: Coherence and Quantum Optics, pp. 495-509. Plenum, New York (1978). L. Mandel and E. Wolf (eds.)

82. Milonni, P.W.: Foundations of Radiation Theory and Quantum Electrodynamics, p. 15. Plenum, New York (1980). A.O. Barut (ed.)

83. Mannheim, P.D.: Classical spin and its quantization. Phys. Rev. D 32(4), 898 (1985)

84. Yao, W.M., et al. (Particle Data Group): J. Phys. G 33, 37-83 (2006). Also see pp. 598 and 968. doi:10.1088/0954-3899/33/1/001 
85. Amsler, C., et al. (Particle Data Group): Phys. Lett. B 667, 1 (2008). 2009 partial update for the 2010 edition (URL: http://pdg.lbl.gov)

86. Akhmetshin, R.R., et al.: Phys. Lett. B 489, 125 (2000)

87. Antonelli, A., et al.: Phys. Lett. B 212, 133 (1988)

88. Fukui, S., et al.: Phys. Lett. B 202, 441 (1988)

89. Wesson, P.S.: Astrophys. J. 394, 19 (1992)

90. Overduin, J.M., Wesson, P.S.: Kaluza-Klein gravity. Phys. Rep. 283, 303-380 (1997). gr-qc/9805018

91. Wesson, P.S.: The cosmological constant problem and Kaluza-Klein theory. Int. J. Mod. Phys. D 10, 905-912 (2001). gr-qc/0104045

92. Wesson, P.S.: Is mass quantized? Mod. Phys. Lett. A 19, 1995-2000 (2004). gr-qc/0309100

93. Wesson, P.S.: The equivalence principle as a probe for higher dimensions. Int. J. Mod. Phys. D 14, 2315-2318 (2005). gr-qc/0601065

94. Wesson, P.: Wave mechanics and general relativity: a rapprochement. Gen. Relativ. Gravit. 38, 937-944 (2006). gr-qc/0601059

95. Overduin, J.M.: Solar system tests of the equivalence principle and constraints on higher-dimensional gravity. Phys. Rev. D 62, 102001 (2000). gr-qc/0007047

96. Biesiada, M., Malec, B.: White dwarf constraints on exotic physics. Phys. Rev. D 65, 043008 (2002). astro-ph/0109545

97. Biesiada, M., Malec, B.: Astrophys. Space Sci. 283(4) (2003)

98. Green, M.B., Schwarz, J.H.: Nucl. Phys. B 181, 502 (1981)

99. Green, M.B., Schwarz, J.H.: Nucl. Phys. B 198, 441 (1982)

100. Kaku, M.: Introduction to Superstrings. Springer, New York (1988)

101. Besançon, M.: Looking for extra-dimensions at the weak scale: experimental search for Kaluza-Klein states signatures at the e+e-linear collider. hep-ph/9909364

102. Besançon, M.: Experimental signatures of extra-dimensions at colliders. Acta Phys. Pol. B 36(11), 3487 (2005) (with numerous references). In: XXIX International Conference of Theoretical Physics Matter to the Deepest

103. Grzadkowski, B., Wudka, J.: Light excitations in 5-dimensional gauge theories. Acta Phys. Pol. B 36(11), 3523 (2005). In: XXIX International Conference of Theoretical Physics Matter to the Deepest

104. Wudka, J.: A short course in effective Lagrangians. AIP Conf. Proc. 531, 81-101 (2000). hep-ph/0002180. Lectures delivered at the VII Mexican Workshop on Particles and Fields, Merida, Yucatan, Mexico, 10-17 November (1999)

105. Ashtekar, A.: Lectures on Non-Perturbative Canonical Gravity. Singapore, World Scientific (1991) (with R.S. Tate) 